\title{
How Artists Can Develop Their Artwork, Its Market and Deliver Their Creative Content for Virtual Reality Environments?
}

\author{
Maureen Kendal \\ Ravensbourne University \\ London, UK \\ maureen@dreamstudio.io
}

\author{
Mehmet Mulla \\ Dreamstudio.io \\ London, UK \\ mehmet.h.mulla@gmail.com
}

\author{
Elaine Thomazi-Freitas \\ London Metropolitan University \\ London, UK \\ e.thomazifreitas@londonmet.ac.uk
}

\begin{abstract}
This research investigates how artists, from traditional fine art and applied art backgrounds, can create innovative immersive and virtual art and build upon their tangible skills, through a digital pipeline and tools? Findings indicated opportunities to co-create partnerships, develop transferable design skills; and exhibitions which enable new markets and audiences.
\end{abstract}

Virtual Reality. Immersive technologies. TiltBrush. Visual effects. Spatial audio.

\section{INTRODUCTION.}

The project aimed to introduce a variety of eXtended Reality (XR) experiences to PingHub network of artists and creatives, to prepare the way for an immersive platform for use by artists, gallery owners, globally dispersed, to enable greater interconnectivity for their audiences, patrons and artists.

The PingHub curatorial team led by Peng Seng Ong, investigated immersive technologies, providers, costs and availability; the chosen provider offered hands-on tasters of Virtual Reality (VR) tools, global partners and an understanding of usability and psychological measures.

The resulting workshop invited exhibiting artists at the PingHub Silk Road exhibition, at the Winns Gallery, London, UK, with artists from the Keeni Kessler Gallery in Penang, Malaysia. Artists collaboratively working with specialists across a development pipeline. Before and after the practical workshop, Artists' panels were invited to live link ups for discussion. Topics included: aesthetics, patronage; funding; methods and pipeline process.

Insights from the artists' explorations, offered new ideas for future steps towards a development of a virtual platform. Considerations included investment and accessibility to immersive technology, 3D graphical, audio and interactive tools and their configuration and insight into how experience of VR technology for artists may find a new home in a virtual immersive setting.

The project aims were to:

1. Introduce immersive design skills to these fine art artists.

2. Enable artists to use these tools independently or in collaborative multi-disciplinary partnerships with VR specialists.

3. Investigate how the transfer of artists' creative content to a VR platform could promote their work across PingHub galleries in other locations (UK and globally).

4. Market the virtual immersive work of artists to new audiences and galleries.

Other forms of immersive medium, including Augmented Reality (AR) were considered and will be discussed as these can benefit artwork delivery and its marketing.

\section{METHODS / APPROACH}

To enable collaborative co-design practice between the two galleries, 'MasterPieceVR' was selected as the primary software application. Due to bandwidth restrictions, 'TiltBrush' by Google was used instead. Collaboration was limited to live stream 
discussion between the artists in their respective galleries. Later the VR artwork was compiled into one instance of the software. Music composition and design of soundscapes is an essential factor of interaction within this immersive environment. Not only within the perception of the surrounding ambience, but enhancing the process of a person's own creative experience.

\subsection{Creating the 3D VR environment}

Specialists in 3D modelling and Tilt brush VR created the 3D environment with visual reference to the Pinterest moodboard created by the PingHubVR curatorial and artists' team. This included a warm stone Moorish style courtyard with a gushing sparkling water fountain, wide windows offering panoramic views to expansive landscapes and surrounded by small cavernous artists' studios topped with arched robes. Here a relaxing and intimate space enabled the artists to work immersively within their own arched area and yet view other artists working in their studio spaces.

The design encouraged a juxtaposition of small intimate internal spaces with open expansive gestures depicting the outside. In a sense reflecting artists experience of mark making and object making in an inside space whilst making reference to dynamic and powerful forces of nature or an intensity of human feeling and sensorial experience. Affording moments of 'parallel play' and enabling synchronous response to their fellow artists. The virtual environment can facilitate 'parallel play' in ways not enabled by a tangible physical context (Hudson \& Kendal 2007). The wide windows were pre-designed in the MasterpieceVR environment but in the course of the workshop were unable to be transferred to TiltBrush as the pipeline process had to be changed midway through the workshop.

\subsubsection{Physical vs virtual}

Multiple viewpoints, framing and sizing can have great impact on artwork, especially when the user takes a closer look. This opportunity, can provide the viewer with naturally occurring dynamic visual elements i.e. From different angles at different times of day, light and/or shadow can have an effect on the artwork and small details can become more apparent. Other artworks allow for a tactile experience where the viewers can touch the piece, feeling the textures, imperfections, etc. The use of haptics in VR are not advanced enough but then it can be argued that if your intent is to experience the art in virtual form, it should come with physical parameters. While this can be substituted in a virtual environment (to an extent), sometimes knowing a factor of change is occurring naturally can provide a sense of awe and wonder and begs the question, in which ways is the understanding and appreciation of the artwork enhanced by the immersive environment? While other artworks take a different approach from their inception such as use of Augmented Reality (AR), these are intentionally designed this way to create a level of interactivity. An example of this exists through use of projection mapping in the form of 'TEXT RAIN, 2000' by Camille Utterback and Romy Archituv, which is "as much an expression of its viewers as it is of its creators; it is what the viewer makes of it" (Bolter \& Gromala 2005, p.13).

The purpose of a virtual setting is to allow artists to express their work to simultaneously compliment the physical artwork. AR can enhance the physical appearance as it allows viewers to switch digitally produced elements on or off.

It can be argued that increased use of immersive reality technology might diminish traditional art whereas in fact it enhances it through various media or the postcard effect. The postcard effect allows individuals to view the artwork where they would not normally have the opportunity i.e. Michelangelo's 'Statue of David', is available for viewing in the Galleria dell'Accademia, Florence, Italy. This has in the past inspired/motivated individuals journey to far off lands. Traditional multimedia offers imagery and videos e.g. Silk Road or Vikings. Immersive media offers solutions that bring to life unique customised visual experience, accessed via QR codes or mobile AR software such as Blippar, allowing content to be accessed as a preview or enhanced immersive experiences. Not only are visual aspects enhanced but also composite sounds, and interactivity locate enriched layered 3D experiences.

\subsubsection{Music / spatial audio}

This soundscape is not a mere assemblage of sounds but is to be integrated into the VR environment; it derives from a conscious interpretation of each artist's views on their work, the visual element, space and time. Music becomes the touch on the skin, sometimes the sight, and at others the actual thinking.

The soundscape composition was generated from audio interviews with the artists. They provided a context to their personal and artistic background alongside the cultural context that connected their works. Each narrative translated a unique visual world into sound images and, more importantly, for the expressive component of the human voices. The human voice carries a strong emotional element in a way that words barely capture.

The transition between meaning and emotion from the captured narrative to the abstract soundscape consolidates the proposed approach to the soundscape composition. There is a process of 
translating meaningful sounds and syntax into abstract sounds to be used as discrete elements within the soundscape composition.

\section{OBSERVATIONS}

Observations from the workshop included simulation of original physical works and placing these on pedestals ready for an exhibition, experiments with the sculpt and modelling tools, overlaying, layering, selecting, collage, placements, orchestration, importing textures and $3 \mathrm{~d}$ objects, interacting with other fellow Artists and their work by placing or over laying work in response. Typical side effects of immersion included loss of a sense of time.

\subsection{Survey results (February and March 2019)}

After the workshops ended, participants were asked to complete a short survey asking about their experience. Themes included skill development, collaborative working, resulting art-works, pipelines, platforms, business model for art production and future engagement. Generally well received, fifty seven percent of the participants gave the experience 5 stars with another twenty eight percent giving it 4 stars (Figure 1). The remainder felt they needed more time and understanding to give a more appropriate response.

Overall, the majority were extremely interested in future workshops and described the experience as innovative and productive (Figure 2). While many had their first experience of VR technology at the workshop, all participants said they 'agree' or 'strongly agree' to the experience being engaging. Furthermore, their responses showed methods of interaction were easy to learn and use, this included navigation:

The barriers are obvious - lack of the requisite tech skillset however the possibilities of thinking planning and delivering an artwork were hugely interesting to me and I have been pursuing this aspect of my practice ever since - Anonymous feedback from survey

Although the interface and experience is not too difficult for beginners, ease of use and access is likely attributed to having an experienced VR artist and support team available to pre induct participants into the controls and the experience. Usability and accessibility is enhanced by encouraging internal cognitive model:

A user attains a more accurate internal model of the system through practice - through an ongoing cognitive processing of the external complexity of the system. (McGinity 2018).
Please rate your overall experience of the workshop ( 5 stars being the highest)

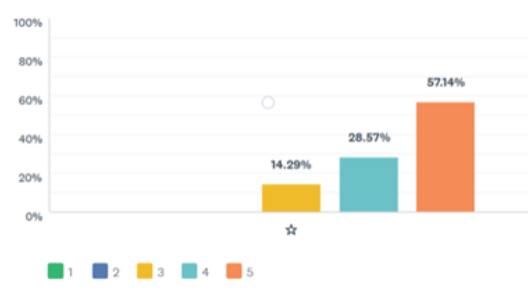

Figure 1: Survey results (Graph of overall experience).

How would you describe your experience in the VR environment (please select all that apply)

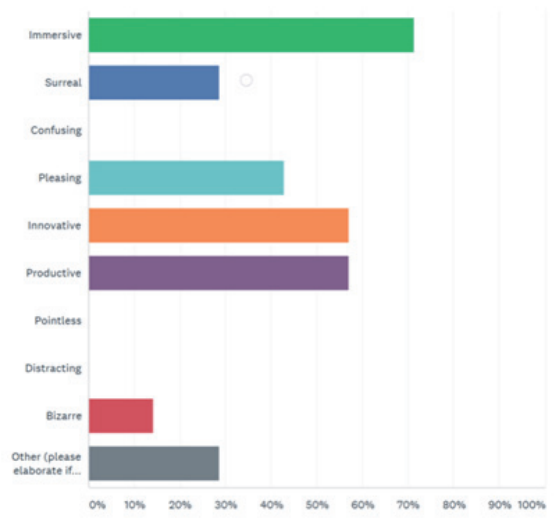

Figure 2: Survey results (Graph describing experience in VR environment.

While the integration of sound / audio is a crucial aspect of the experience, on the original VR experience, the artists did not listen to a soundscape. Traditionally, audio is used as an interactive marker. However, in this project music creation is conceived with a close identification to film music language: the sounds were not purely musical, they generated an underscore, a meaningful soundscape inside the VR environment.

\subsection{Analysis}

\subsubsection{Remediation}

Artists' used this immersive experience and the VR pipeline for remediation and/or innovation. "Remediation is the making of new media forms out of older ones" (Bolter \& Gromala 2005, p.83). In 'Age of Exploration, Slaves to Silk' (2018), an exhibited physical art work by Fion Gunn, is made of materials including silk, acrylic and mixed media and presented without the addition of elements such as lighting, sound or a physical substance such as water, just as the artist intended. 


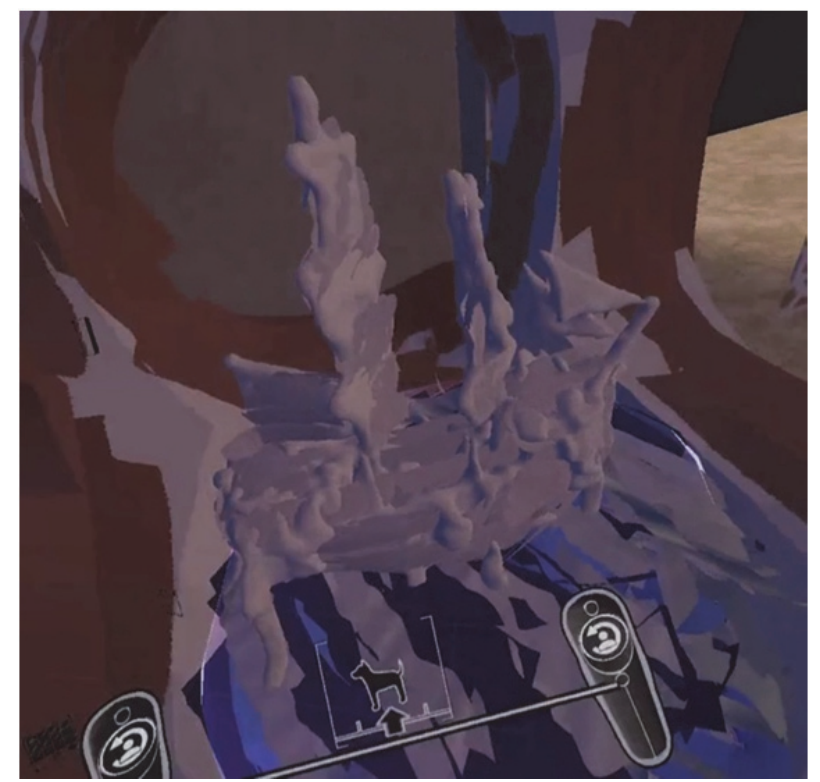

Figure 3: 3D scanned VR model of 'Age of Exploration, Slaves to Silk', 2018 by Fion Gunn.

By creating a virtualised version of the artwork by means of 3D scanner (Figure 3), the opportunity to introduce such elements not only allow the artwork to be presented in a dynamic instance but also avoidance of damage to the artwork thereby changing the parameters of its perception and allow it to be experienced entirely differently. "...Creating a VR environment gives it another story. There's another narrative going on in that work which separates it from the original material artwork." Furthermore, through the process Fion was able to create a $3 \mathrm{D}$ model of herself through use of the 3D scanner, which was placed in the virtual model. Through this process artists can portray themselves and their models, as virtual and or visceral characters within the online world. Art can transmit humour and allegory through puns, parody and poetry includes biting social satire, e.g. the artist Carla Gianna's use of an Artificial Intelligence $(\mathrm{Al})$ persona to reflect satire.

The spectator can, to an extent, identify with the artist and the 3D character of herself and walk around the $3 \mathrm{~d}$ model to gain 360 -degree views. For many of the artists, 3D camera scans were created and subsequently cleaned up. Sometimes objects were enhanced using TiltBrush effects e.g. simulation of water. Thereby adding virtual elements to the original concept of a physical artwork.

\subsubsection{Innovation}

Some artists understood that their traditional design process could not be used and sought to create completely new working methods.

Doris Hafner, artist:

"The process is different in the VR where you do not carve in to a stone block, but create the shape directly."

\subsubsection{Abstraction and reproduction}

Once an art piece can be digitally reproduced, there are countless ways to generate art. Digital design decisions based around abstraction, reproduction and repetition of an artwork can generate aesthetic, elegance, rhythm, and variation.

Paul James, 3D specialist, developed a texture palette, with unique variation:

$$
\begin{aligned}
& \text { "I was in the woods the other day and took } \\
& \text { scans of this stump in the middle of the forest, I } \\
& \text { can come back in now and put that in a different } \\
& \text { program, makes a 3D scan all textured for you, } \\
& \text { you clean up the mesh and you can import it into } \\
& \text { the game engine for a real top notch game asset } \\
& \text { for really very little work." }
\end{aligned}
$$

Likewise, as stated in methods, audio elements were abstracted and reproduced. A collage of sounds was layered with improvised melodic fragments. The resulting soundscape illustrated a model of possibilities. The use of spatial sound in VR is quite specific and the boundaries are unknown. Consider cutting edge Imogen Heap's VR concert developed with TheWaveVR in 2018 (Deahl 2018). VR expands how we listen to and perceive music as an entirely different representation of sound. AMBEO 3D Audio Technology from Sennheiser translates this distinction based in ambisonics, a system for capturing or representing a spherical sound field (Sennheiser 2019).

\subsubsection{Collaboration}

Artists gained insight from working with other artists and specialists within the VR pipeline

Yohjan Salamanca, student:

“...A general knowledge of everything plus your expert knowledge on your own subject is very useful and it can open up just more possibilities and it will allow you to explore your work but also other people's work". 


\subsubsection{Markets}

How can artworks be purchased within the immersive worlds? Often the sale of an artwork is encouraged when the purchaser or patron feels a connection with the artist, their creative process or a sense of common experience or sensibility. In medium and high-end markets, the artwork is perceived as an investment, with aesthetic and emotional connection as secondary. The users include artists generating user-generated content, gallery owners and potential purchasers. Different users will require different interface requirements. Participants may buy into an event, and/or the purchase of digital limited collectable and/or physical artwork. The business model for immersive Art world needs to be mapped and trialled to test economic appetite. However investment and resourcing is substantial including professional costs, copyright, production fees, specialist equipment, spatial audio, field visual and audio recording.

\section{Alan Hudson, Virtual Worlds designer:}

\begin{abstract}
"Maybe one of the problems with Second Life is that you've got to appeal to both markets, you've got appeal to people who are just going in there to experience things and other people doing serious building and they'll have very different setups"
\end{abstract}

\section{Peng Seng Ong, PingHub Founder:}

\footnotetext{
"...It also depends on the volume of sales, in some sense it's no different than if the artist was to produce a limited edition of their painting"
}

\subsubsection{Performance}

Artistic practice using immersive graphic tools like 'TiltBrush' enable performative movement at a human scale. Here the digital artist is not bent over a laptop, articulating visual design elements as if inside a small 2D screen. They can move through real physical 3D space on a human scale and generate a performative artistic practice, creating $3 \mathrm{D}$ objects and environments, combining a range of artistic practices from tangible, digital and virtual mark-making. In the virtual world, 3D immersion simulates the artist's physical and performative engagement, to draw and paint using the full extent of the arm pitted from the torso, balanced by the hips. Moreover the sculpt and model tools offer a culpable physicality. The notable abstract expressionist painter Jackson Pollock's 'action painting', reminds us how an artist can use force of their whole body to paint. For Pollock it was a frenetic dancing style. For our artists, using Immersive Tools to create 2/D and 3D art works, facilitated their almost free flowing movement in space. To the real-world observer, each artist stands as a full figure with head set 'dancing' as if in an improvised choreography, focused intently and in flow.

\subsubsection{Affordance}

The functions of a device that allow use and access of an immersive environment should be effortless, not just for the creator but also for the audience who wish to experience it. Even though our users are participants rather than competitive game players, their activity in TiltBrush is immersion into a rule-bound world, despite giving the external impression of freedom of movement. The challenge for the artist is how to access and learn the complexity of available graphical, paint and sculpt tools, developing an internalised model of the VR tools and environment in order to gain a sense of actual control and external mastery.

Affordance needs to take on additional meaning for artists, as they are more hands on in the creation process. The interface of Tilt Brush does just this as it draws inspiration from the techniques of traditional art by having the right controllers (associated with the headset) be used to interact with the environment, much like a physical art brush. The left controller provides all the features required to produce the work, like an old art palette. "Signifiers communicate where the action should take place" (Norman 2013, p.14). Thus the level of acceptability is increased and draws from the concept of affordance. These functions provide the necessary mode to interact appropriate to the scenario.

\section{CONCLUSION}

This workshop project identified these considerations:

- Artists' contribution to how it feels to be human and an avatar within virtual world space.

- Concerns around online surveillance and its opportunities for online empowerment.

- Modelling of distribution access to new audiences and re-imaginings.

- A range of viewpoints within this $3 D-360$ degrees constructed space, affording both close up intimate frames and the wide-long view.

- Artists as digital actors e.g. by placing a 3D model of the artist within their own construction / object / environment or by designing an avatar to resemble an alternative self.

- Artist with headset, as dancing figure, in performative practice.

- Combination of tangible, digital and 'virtual world' production to create new hybrid processes. 
For game-play, the player's agency and the relational schema of interaction needs to be finely balanced to achieve immersion and a reinforced sense of internal mapping and modelling. Balance of a rule-bound world with perceived randomness and user agency is necessary for an interactive immersive environment.

Development of the Pinghub platform can implement narrative arcs to enable user engagement. Artists' creative flow and process can include cycles of deep reflection to re-design, remediate and/or innovate deep structures and elegant design.

In this workshop, aesthetic decision-making is a collaboration between artists and specialists. Specialist skills in immersive / interactive arts, composition and experimental music are required to develop VR immersive platform. All participants gained from this opportunity to co-design with these specialist teams using different design languages; and through this pipeline, integrate their work within their art practice.

Future development to build an interactive immersive virtual environment for PingHub and creative hubs can build on Artists' experiments and considerations during and after the workshop. Moreover the design of interactivity, game-play, narrative arcs and usability can enrich outcomes of engagement, affordance, market outreach, collaborative benefits, design methods around abstraction and reproduction, and performance.

\section{REFERENCES}

Bolter, J. D. and Gromala, D. (2005) Windows and Mirrors: Interaction Design, Digital Art, and the Myth of Transparency. MIT Press, Cambridge, MA.

Csikszentmihalyi, M. and Csikszentmihalyi I. (1990) Flow: The Psychology of Optimal Experience. Harper and Row, New York.

Deahl (2018) Inside Imogen Heap's cutting-edge VR concert, in The Future of Music with Dani Deahl, S1E1.

https://www.youtube.com/watch?v=HoDqeunBH10 \&list=PL39u5ZEfYDEMiTaRs-5xsUB5YP8sCo0P\&index=2\&t=0s (retrieved 10 April 2019).

Giannini, T. and Bowen, J. (2018) Of Museums and Digital Culture, A landscape View, EVA London 2018. https://dx.doi.org/10.14236/ewic/EVA2018.34 (retrieved 10 April 2019).

Kendal, M. and Hudson, A. (2011) On the Beach A handbook for using $3 \mathrm{D}$ virtual digital platforms like Second Life - 'the WEISL' - 'Writing Explorations in Second Life. EVA London 2011. https://ewic.bcs.org/content/ConWebDoc/40570 (retrieved 10 April 2019).

Norman, D. (2013) The Design of Everyday Things. MIT Press, Cambridge, MA.

McGinity, M. (2018) La Dispersion Du Flls: Into regions of incomprehensibility. EVA London 2018. https://dx.doi.org/10.14236/ewic/EVA2018.64 (retrieved 10 April 2019).

Propp, V. (1928) Morphology of the Tale. Leningrad.

Sennheiser (2019) Capturing in Ambisonics, in AMBEO for Virtual Reality. https://enuk.sennheiser.com/ambeo-blueprints-virtual-reality (retrieved 10 April 2019). 